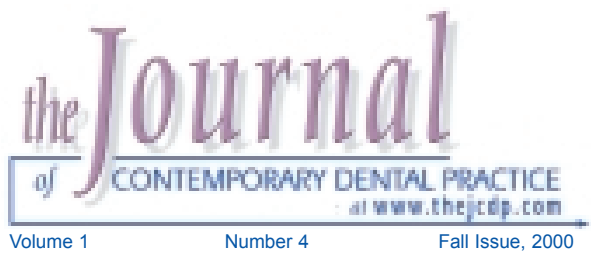

\title{
Anticalculus Effects of a Novel, Dual-Phase Polypyrophosphate Dentifrice: Chemical Basis, Mechanism, and Clinical Response
}

\section{Donald J. White, PhD; Robert W. Gerlach, DDS, MPH}

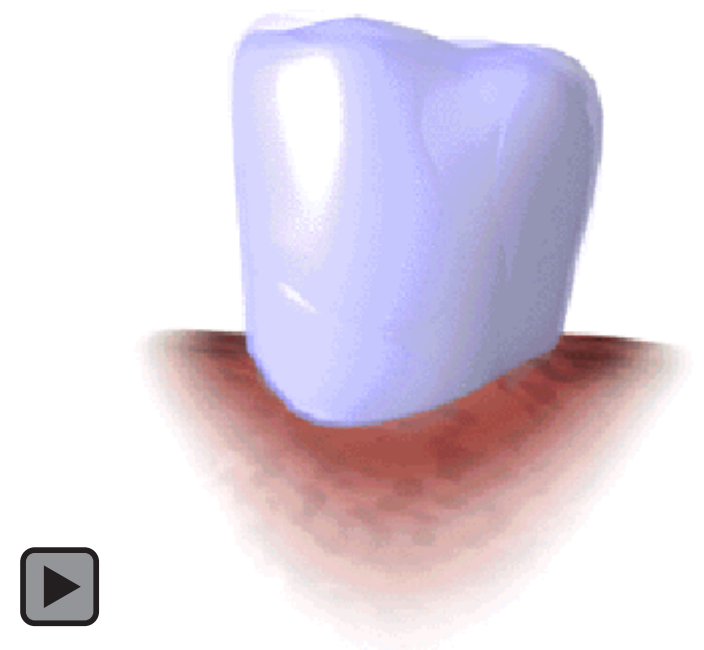

Abstract

A primary patient motivation for oral hygiene is effective cleaning. Dentifrice serves this function by including ingredients such as abrasives, surfactants, and specialized cleaning ingredients such as anticalculus agents. This introductory article aims to introduce professionals, educators, and researchers on the rationale behind the development of an improved cleaning dentifrice formulation, Crest $^{\circledR}$ Multicare Advanced Cleaning. This new dentifrice is based upon the application of an improved tartar control/cleaning ingredient that is a polymeric adjunct of a pyrophosphate anion commonly applied in tartar control and stain control whitening dentifrices. The polypyrophosphate anion, also referred to as sodium hexametaphosphate, produces superior activity and substantivity on oral surfaces as compared to both pyrophosphate and some other commonly used dental cleaning ingredients and cleaning/conditioning adjuncts. The increased activity and substantivity translate into significant improvements in the prevention of dental stains and supragingival calculus and in the non-abrasive removal of dental stains.

This article describes the structure of polypyrophosphate as compared to the parent pyrophosphate molecule, the rationale for its improved chemistry, and, in particular, its tartar control chemistry. In addition, the fundamental mechanisms of calculus formation and inhibition are

reviewed. Lastly, a preliminary clinical study evaluating the improved efficacy of a polypyrophosphate dentifrice is described where the tartar control activity of the polypyrophosphate dentifrice is shown to be superior to that of a clinically established and marketed industry standard pyrophosphate dentifrice.

Keywords: Tartar, calculus, oral hygiene, polyphosphate, polypyrophosphate, clinical trial, hexametaphosphate, dentifrice, tooth stain, mouth feel, conditioning, substantivity 


\section{Introduction}

The formation and removal of dental calculus poses a significant problem for many adult patients and dental professionals. ${ }^{1,2}$ Clinical and epidemiological surveys confirm significant calculus formation in a broad portion of the population with prevalence approaching $90 \% .^{3}$ In addition to the obvious cosmetic implications (Figure 1), supragingival calculus may impede normal hygiene (particularly flossing), and in excessive quantities, may contribute to gingival recession. ${ }^{3}$

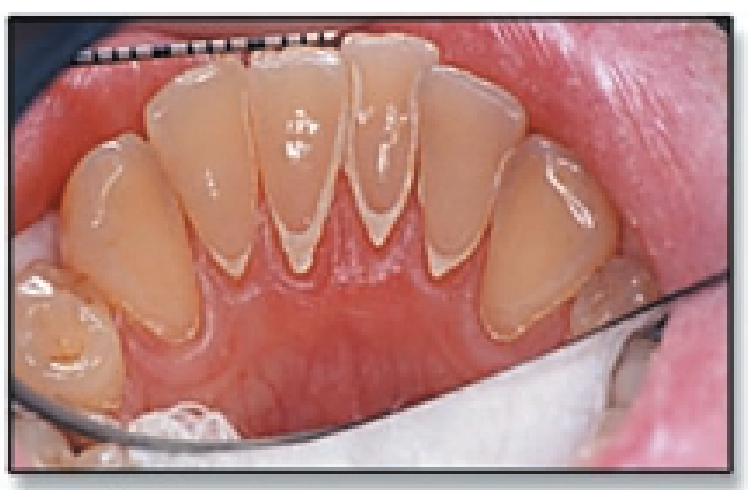

Figure 1.

Subgingival calculus is more commonly associated with chronic periodontal diseases, although debate continues as to whether these deposits are the cause, promoter, or result of the inflammatory processes. ${ }^{1}$ Once formed, calculus removal can only readily be accomplished through time-consuming scaling often in areas that are difficult to visualize and/or access.

While removal usually necessitates mechanical debridement, calculus deposition may be readily prevented by topical application of various chemical inhibitors such as those found in the socalled "tartar control" toothpastes. The first clinically proven tartar control dentifrice $\left(\right.$ Crest $^{\oplus}$ Tartar Control, The Procter \& Gamble Co., Cincinnati, OH US), which was introduced in 1985, contained sodium pyrophosphate as the anticalculus ingredient. ${ }^{4,5}$ In this dentifrice, pyrophosphate was combined with sodium fluoride for anticaries effects in a silica abrasive base. Today, tartar control ingredients are found in more than $50 \%$ of all dentifrice sold in the United States. Extensive clinical research has confirmed the efficacy of these formulations for both calculus and caries prevention. ${ }^{2}$ In addition, tartar control ingredients are combined in virtually all of the so-called "multibenefit" toothpastes. Tartar control ingredients are thus found in commercial formulations offering whitening (stain removal), plaque prevention, the control of gingivitis, and more recently the treatment and prevention of dentinal hypersensitivity.

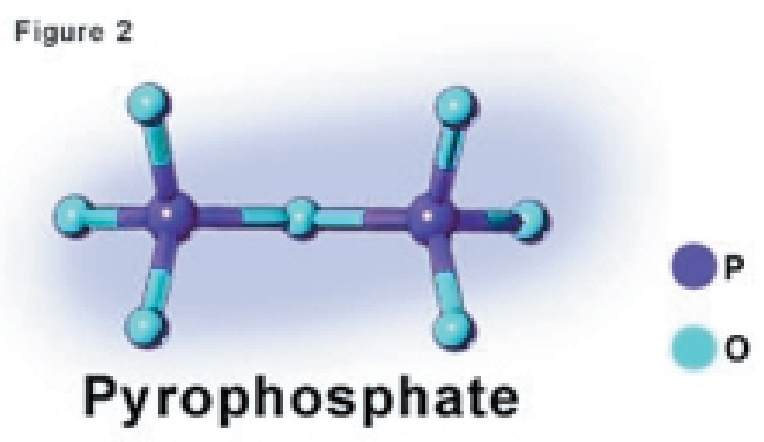

Figure 2 illustrates the chemical structure of pyrophosphate, the first widely-marketed anticalculus ingredient. In its role as a mineral chelator/mineralization inhibitor, pyrophosphate helps naturally regulate mineralization with effects in both bone/tooth formation, calcium homeostasis, and in ectopic mineralization disorders. ${ }^{6}$ The primary feature of pyrophosphate and related molecules that contributes to the clinical response is the strong binding interaction with calcium, both in solution and on surfaces (Figure 3).

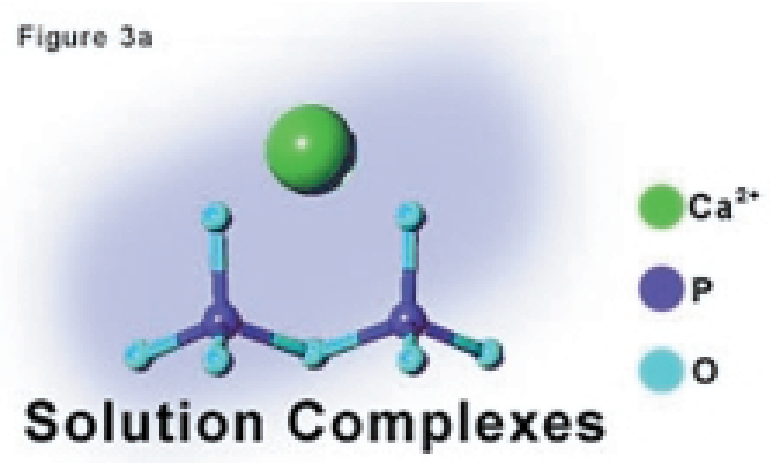

Pyrophosphate interacts with calcium by virtue of ion-pairing in solution and at the surface with cationic positive charge of the calcium attracted to the negative charges of oxygen on the phosphate anions. 


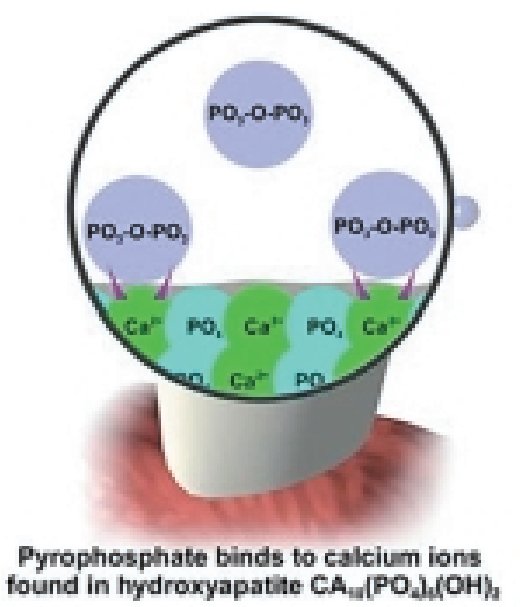

Figure 3

Although the mechanisms of calculus formation and prevention are still under investigation, the fundamental processes have been characterized. Tartar formation is foremost the result of mineralization of dental plaque biofilms. ${ }^{7}$ Soft bacterial plaque mineralizes until these deposits literally petrify, after which the remarkable hardness and tenacity inhibits removal during routine personal oral hygiene. Figure 4 illustrates this process wherein the acquisition of dental pellicle films, the deposition of plaque, and the subsequent mineralization and coalescence of mineral within plaque form dental calculus. ${ }^{7}$
The mineral in supragingival dental calculus is comprised of calcium phosphate in crystal phases of differing density, shape, surface area, and aqueous solubility. Mature calculus mineral begins to resemble the mineral in tooth enamel and calcium hydroxyapatite. ${ }^{8}$ This similarity hinders tartar control through calculus dissolution since etching solutions effective against calculus should damage enamel as well. The antitartar activity of pyrophosphates and other mineralization inhibitor molecules is achieved primarily through the control of the mineral formation within dental plaque. On the microscopic level, the growing/hardening crystal surfaces acquire calcium and phosphate from ions in solution.

Like children's building blocks, these ions are assembled into a regular pattern which defines the solid 'crystal.' (The ions lose their 'waters of hydration' upon crystallization as well.) The mineralization inhibitor ions can bind some of these ions in solution, never allowing crystal growth. However, this latter effect is short lived and is largely lost several minutes after toothbrushing. Even more importantly, pyrophosphate and like inhibitors can bind to the mineral surfaces. In these locations, pyrophosphate can disrupt the mineral building process primarily because the structure does not
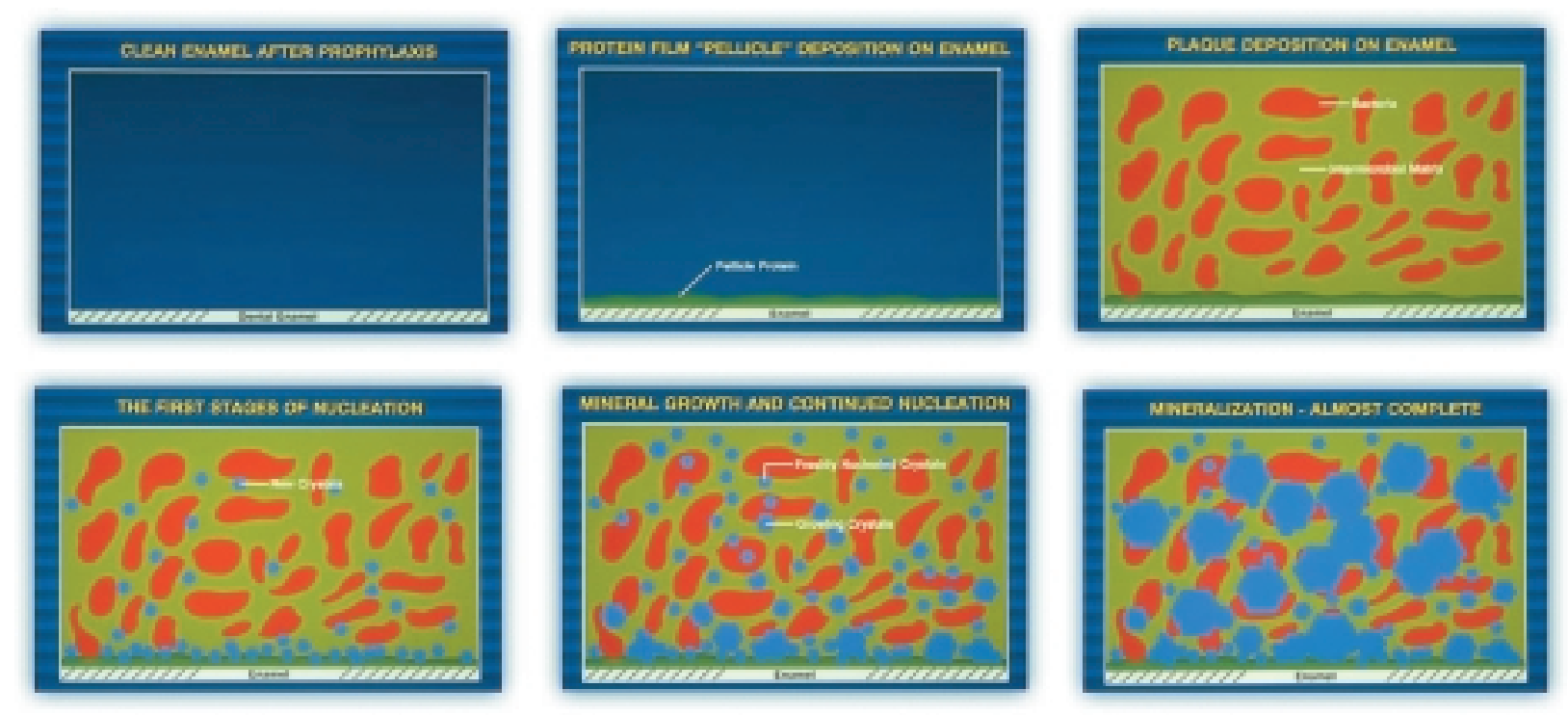

Figure 4 

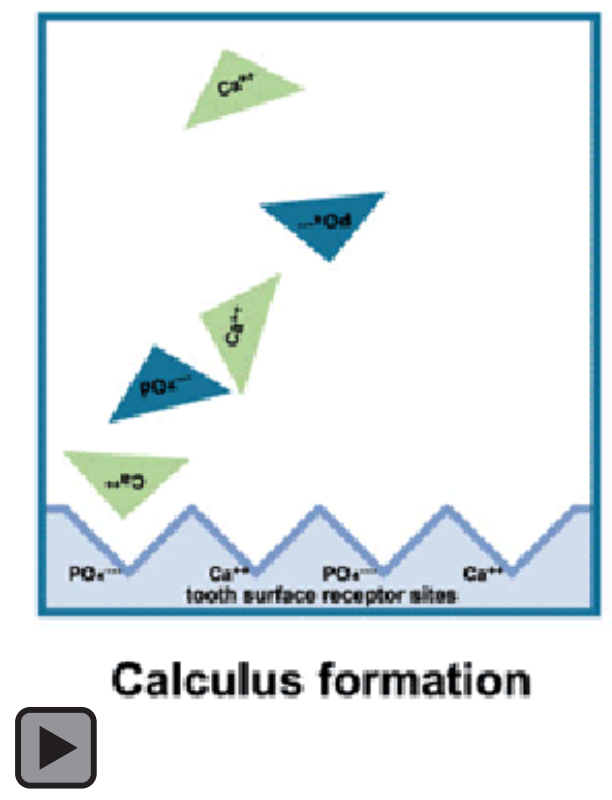

Figure 5

adequately fit the developing mineral lattice. With the reaction energetics appropriately compromised, the crystal does not grow and remains immature. The effects of pyrophosphates and like inhibitors at disrupting crystal growth through surface actions occur at quite low concentrations. Research supports that these ingredients are retained in effective concentrations within dental plaque. ${ }^{10}$

Figure 6 illustrates the consequences of the chemical actions of mineralization inhibitors on actual tartar prevention. ${ }^{9}$
These inhibitors enter plaque with toothbrushing, bind to existing crystal surfaces, and leave substantive inhibitors behind within plaque fluid in intermicrobial matrix. The inhibitor on crystal surfaces stops crystal formation, while importantly, the substantive inhibitor prevents nucleation or crystal growth. In net, pyrophosphate and like molecules function by acting as mineralization inhibitors essentially controlling plaque solution chemistry and mineralization processes. Such agents do not dissolve or remove calculus, but instead prevent plaque from becoming cementlike due to mineralization. Soft plaque can be removed with regular brushing. For these reasons, patients who regularly use tartar control toothpastes experience significant clinical reductions in tartar formation.

Pyrophosphate was the first and is one of the most common ingredients used for dental calculus control. Clinical studies have shown clinical efficacy for pyrophosphate formulations in reducing the development of calculus between dental prophylaxes ranging between $20-40 \%$. $1,11-13$ Clinical response is most commonly measured in terms of tooth surface area coverage using a standard method such as the Volpe-Manhold Index (V-MI). ${ }^{14}$

While safe and highly effective, pyrophosphate salts have some limitations. First, these agents do not completely inhibit calculus formation in most individuals. Several factors limit efficacy including the substantivity of the active form and subsequent sustained actions. Once adsorbed at
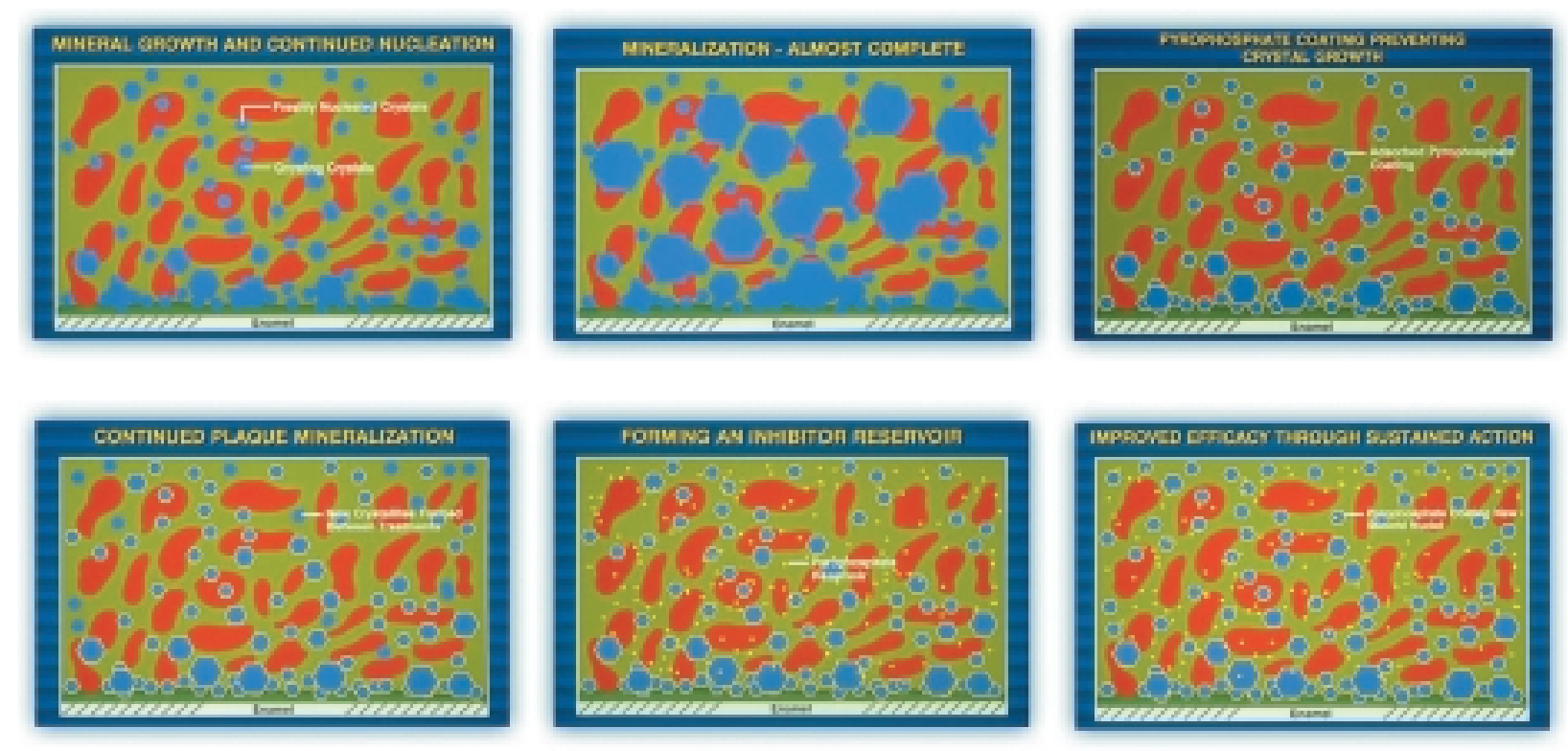

Figure 6

The Journal of Contemporary Dental Practice, Volume 1, No. 4, Fall Issue, 2000 
tooth or calculus nucleation surface sites, pyrophosphates can be deactivated either through desorption (promoted by re-adsorbing salivary proteins) or hydrolysis. Hydrolysis can result from either enzymatic (phosphatase) or aqueous degradation..$^{15}$ Both processes limit the lifetime of the parent inhibitor molecule and, hence, the effective substantivity of the molecule.

One means to overcome the limitations of pyrophosphate salts as intraoral cleaners is to apply higher molecular weight analogues, the polymeric phosphates, which are used widely in household and industrial cleaning formulations because of their safety profile and superior activity relative to lower molecular weight pyrophosphate analogues. These molecules called by various names in the chemical and trade literature including condensed phosphates, glassy phosphates, sodium hexametaphosphates, etc., are prepared from the condensation polymerization of lower molecular weight phosphates at high temperatures. Figure 7 compares the structure of linear polymeric phosphates to various phosphates.

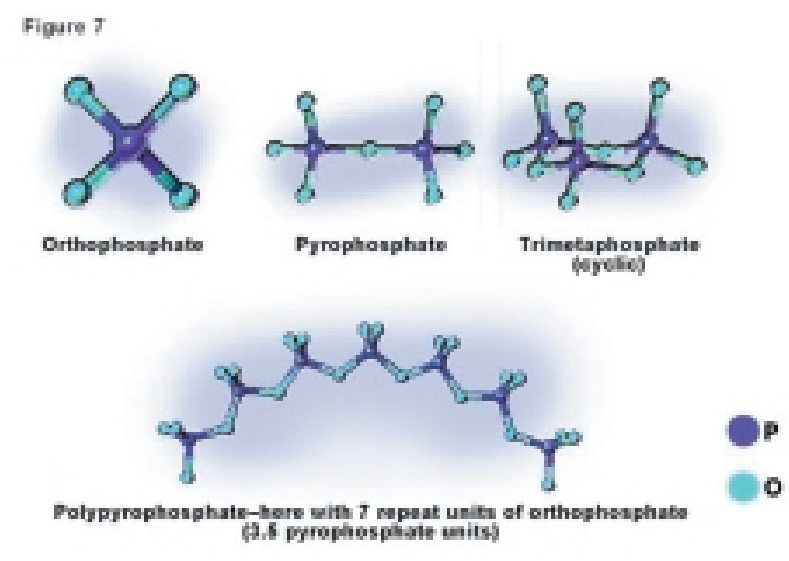

These structural differences contribute to the different chemical properties of these anticalculus agents (Table 1).
Our laboratory has generated a significant chemistry profile documenting surface and solution physical chemical activity of polypyrophosphates which recommend these molecules as important upgrades for dentifrice formulations. This research demonstrates greater surface affinity of polypyrophosphates to calcium hydroxyapatite minerals than pyrophosphate. ${ }^{16,17}$ The improved adsorption results from multiple binding sites that limit salivary protein desorption of polypyrophosphate compared to pyrophosphate, thereby, increasing potential retention and substantivity of the polypyrophosphates. The high surface charge generated by polypyrophosphate produces significant benefits in preventing the secondary adsorption of stain chromagens, hence, providing a means for chemical rather than abrasive tooth stain control. ${ }^{18}$ In addition, the polypyrophosphates resist hydrolytic deactivation by plaque and saliva phosphatase enzymes with reaction products including active inhibitor species.

Footnote: "While highly effective between brushings intraorally, polypyrophosphates in conventional dentifrice formulations maintain stability only for a matter of weeks."

While the superior solution and surface chemistry of polypyrophosphates (as opposed to pyrophosphate) are well known, applications of higher chain polyphosphates in toothpastes have been limited by stability considerations, most particularly the long term hydrolytic stability of these polymers in the aqueous phase of conventional dentifrices. ${ }^{*}$ Importantly, the advent of cost effective and functional, dual-phase dentifrice packaging has opened the possibility for applying polypyrophosphate ingredients for oral care applications. ${ }^{19}$

Table 1

\begin{tabular}{|l|c|c|}
\hline Characteristic & Polypyrophosphate & Pyrophosphate \\
\hline Average P atoms in polymer & 21 & 2 \\
\hline Molecular Weight of Anion & 1500 & 174 \\
\hline Calcium Binding Solution & $>>$ & \\
\hline Calcium Apatite Surface Binding & $>>$ & \\
\hline Stability in Water & $* *$ & $>>$ \\
\hline
\end{tabular}

${ }^{* *}$ Rationale for dual phase formulation 
Our fundamental research on polypyrophosphate coupled with modern packaging innovation has enabled development of a stable and effective polypyrophosphate dentifrice. These products are marketed as $\mathrm{Crest}^{\circledR}$ Multicare Advanced Cleaning Formula in the United States and will soon be available in Europe as Blend-a-Med Mediclean and AZ Ultraclean in Europe. The considerable physical chemistry and clinical research supporting the mechanism of action and effectiveness of polypyrophosphate is in preparation for publication which will occur over the next year. The remainder of this paper describes preliminary tartar control clinical testing examining the efficacy of a polypyrophosphate dentifrice compared with industry standard pyrophosphate dentifrice control demonstrating the superior clinical potential of this novel ingredient.

\section{Methods}

A randomized, double-blind, multicenter clinical study compared efficacy and tolerability of polypyrophosphate-containing dentifrices to a positive control. This study was conducted in two phases over a 10-week period.

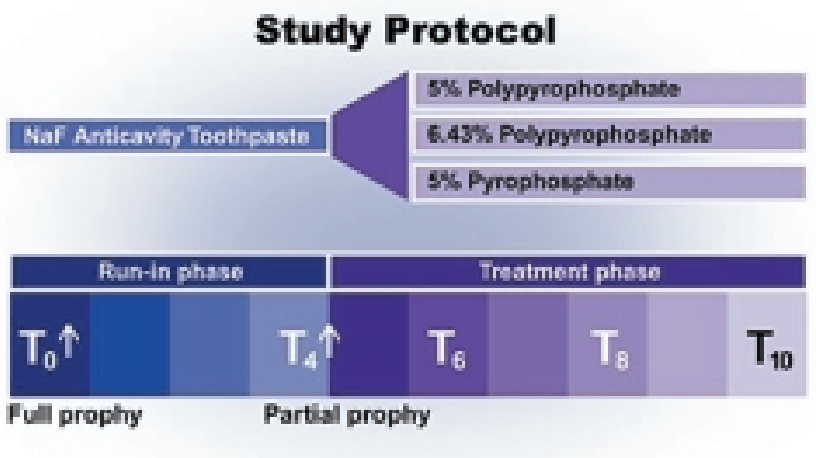

Following a 4-week run-in to assess supragingival calculus formation, eligible subjects were randomized to one of three dentifrice treatment groups and then efficacy and tolerability were evaluated over a 6-week treatment period using blinded methods. The three treatment groups were:

- Experimental, dual phase, polypyrophosphate tartar control dentifrice containing $7 \%$ sodium polypyrophosphate ( $5 \%$ as anion)
- Experimental, dual phase, polypyrophosphate tartar control dentifrice containing 9\% sodium polypyrophosphate $(6.43 \%$ as anion)

- Marketed, sodium fluoride tartar control dentifrice containing $5 \%$ pyrophosphate (as anion) which served as the positive control

The study population consisted of generally healthy employed adult volunteers who provided informed consent and met minimal entrance criteria (Table 2).

\section{Table 2. Study Entrance Criteria}

Inclusion Criteria

- Give written informed consent to participation

- Be at least 18 years of age

- Have a minimum of $5 \mathrm{VMI}$ mandibular anterior teeth

- Agree to refrain from using oral care products other than those assigned as part of the research

\section{Exclusion Criteria}

- Self reported sensitivity to tartar control pyrophosphate containing dentifrices

- Fixed orthodontic appliances on the lower VMI teeth

- Any diseases or conditions which might interfere with examinations or subject safety

The research was approved a priori and overseen by an institutional review board. During the qualification phase volunteers received a thorough dental prophylaxis and then were provided with a regular anticavity dentifrice containing $0.243 \%$ sodium fluoride (Crest ${ }^{\circledR}$ Regular Paste, The Procter \& Gamble Co. Cincinnati, OH USA). Volunteers were instructed to use a specified brushing regimen during this 4week period to accelerate calculus formation. During the run-in period, all brushing was unsupervised and open-label. At the end of the 4 week run-in period, supragingival calculus levels were evaluated and individuals who formed sufficient supragingival calculus using the partial non-brushing regimen were eligible for the treatment phase of the study (Figure 9). 


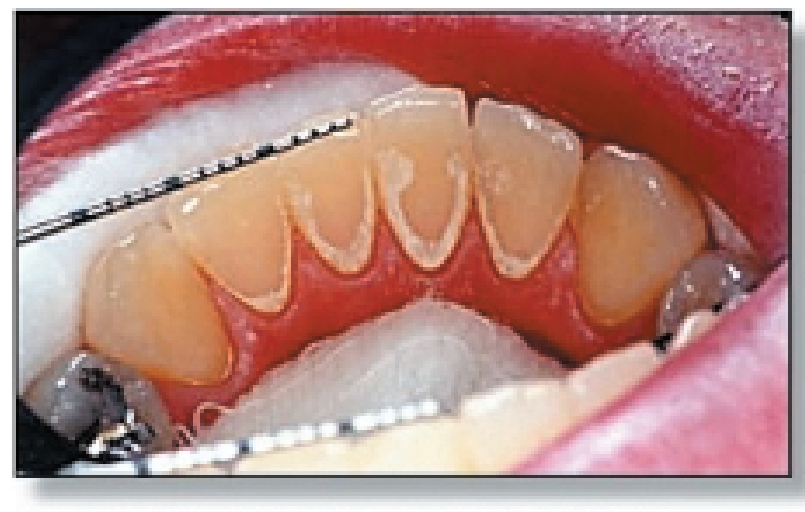

Figure 9. Calculus formation using the partial non-brushing regimen

At the start of the test phase, subjects were provided with a partial dental prophylaxis involving the V-MI teeth and a safety assessment was again carried out. Subjects were then randomly assigned to treatments. Subjects brushed unsupervised under treatment for six weeks with clinical examination of soft tissue safety and calculus at weeks 2, 4 and 6.In the treatment phase, two variants of a polypyrophosphate experimental dentifrice were compared to a marketed tartar control dentifrice formulation with proven clinical efficacy. To assure blinding, all test dentifrices, including the marketed, single phase antitartar toothpaste, were packaged identically in dual phase $3.5 \mathrm{oz}$. pump dispensers, each of which contained 100 grams of the assigned dentifrice. Test dentifrice was overpackaged in a kit along with a soft adult toothbrush (Oral B 40 Soft, Oral B Laboratories, Belmont, CA USA), a 30 second sand timer, and an instruction sheet for use throughout the 6week treatment phase of the study (see Illustration).

To further assure blinding, kits were identically labeled except for a unique subject identification number so that treatment identity could not be discovered by either of the examiners or by thesubjects anytime throughout the treatment phase of the study.

The general design, which included a run-in to assess calculus formation rates followed by comparative efficacy, is commonplace in antitartar dentifrice studies. However, this study design deviated from conventional models in the use of a modified, partial non-brushing regimen (Table 3). This modified model, which has been previously shown to yield elevated tartar formation levels in a wider range of normal clinical subjects, expands potential study eligibility while reducing the study duration needed to assess efficacy. ${ }^{20}$

Table 3. Partial Non-brushing Regimen to Accelerate Calculus Formation

\section{Weekday Instructions}

- Brush all surfaces of teeth EXCEPT the lingual surfaces of the six mandibular anterior teeth

- Brush at least twice daily for at least 30 seconds, covering the full length of the brush head with dentifrice

- After brushing, swish the dentifrice slurry over all surfaces of the six mandibular anterior teeth for about 15 seconds before expectorating

- Do not floss or use other oral care products

\section{Weekend Instructions}

- Brush ONCE per day, including lingual surfaces of the six mandibular anterior teeth for approximately 15 seconds

- Do not floss or use other oral care products

Efficacy was assessed using a standard clinical method (V-MI) that measures supragingival calculus coverage on the lingual surfaces of the six anterior teeth. ${ }^{14}$ Examinations were conducted by a single trained and calibrated examiner who used a UNC-15 periodontal probe graduated in millimeters and air to facilitate calculus visualization and measurement. Tolerability was assessed by visual examination of the oral cavity and peroral area using a standard dental light, dental mirror, and gauze. The structures examined included the gingiva (free and attached), hard and soft palate, oropharynx/uvula, buccal mucosa, tongue, floor of mouth, labial mucosa, mucobuccal/mucolabial folds, lips, and peroral area.

Data for efficacy evaluations were analyzed by a parametric ANCOVA with treatment and center as factors and baseline V-MI scoring as the covariate. Center by treatment interactions were examined, and because these were found to be non statistically significant $(p<0.10)$, this was deleted from the ANCOVA model. Pairwise treatment comparisons were made at the one 
sided 0.10 significance level via the LSD test. Safety data was analyzed by descriptive analysis including OST related adverse events for all treatment groups.

\section{Results}

A total of 214 generally healthy adults were enrolled during the 4-week run-in period. Of these, 13 were lost to follow-up, 5 failed to develop supragingival calculus during the run-in, and 1 individual failed other entrance criteria. The remaining 195 subjects were randomized to treatment, 193 of whom were considered evaluable by study end. The treatment population was well-balanced with respect to age, gender, and ethnicity (Table 4).

In addition, treatment groups were well-balanced with respect to calculus accumulation during the run-in period (Table 5).

Each of the 3 treatment groups averaged approximately $9 \mathrm{~mm}$ of calculus on the lingual surfaces of the mandibular anterior teeth as measured using the V-MI.

\begin{tabular}{|lcccc|}
\multicolumn{5}{c}{ Table 4. Demographics (Evaluable Subjects) } \\
Parameter & $\begin{array}{c}\text { 5\% Polypyro- } \\
\text { phosphate } \\
\text { (N=64) }\end{array}$ & $\begin{array}{c}\text { 6.4\% Polypryo- } \\
\text { phosphate } \\
\text { (N=64) }\end{array}$ & $\begin{array}{c}\text { Pyrophosphate } \\
\text { Control } \\
\text { (N=65) }\end{array}$ & $\begin{array}{c}\text { Total } \\
(\mathbf{N}=193)\end{array}$ \\
Mean & 4043 & & 40 & 41 \\
SD & 7.6 & 9.2 & 8.1 & 8.4 \\
Range & $26-55$ & $23-61$ & $25-63$ & $23-63$ \\
Sex & & & & \\
Female & $44(69 \%)$ & $45(70 \%)$ & $45(69 \%)$ & $134(69 \%)$ \\
Male & $20(31 \%)$ & $19(30 \%)$ & $20(31 \%)$ & $59(31 \%)$ \\
Race & & & & \\
White & $59(92 \%)$ & $57(89 \%)$ & $58(89 \%)$ & $174(90 \%)$ \\
Black & $3(5 \%)$ & $4(6 \%)$ & $6(9 \%)$ & $17(7 \%)$ \\
Asian American & $2(3 \%)$ & $3(5 \%)$ & $1(2 \%)$ & $6(4 \%)$ \\
\hline
\end{tabular}

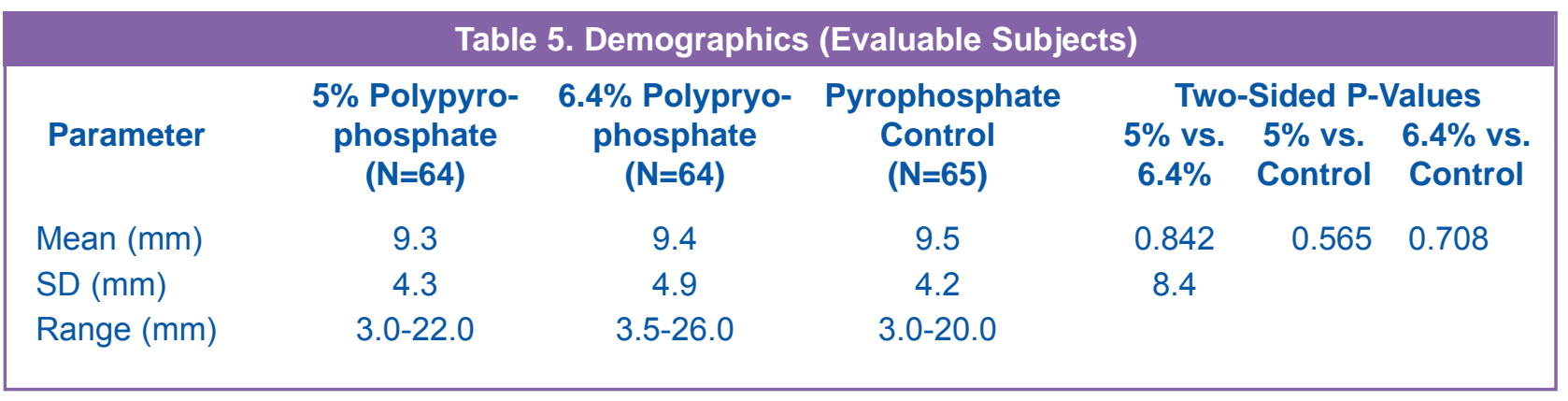


Table 6 summarizes efficacy results from the treatment phase. At weeks 4 and 6 , the mean V$\mathrm{MI}$ score for the $5 \%$ polypyrophosphate tartar control group were statistically significantly (one sided $p$ 0.022) lower than the mean V-Ml score for the marketed antitartar dentifrice. Percent reductions for the latter comparison measured $19.6 \%$ at week 4 and $15.0 \%$ at week 6 . At each test period, the mean V-MI score for the $6.43 \%$ polypyrophosphate tartar control dentifrice group was statistically significantly (one sided p 0.040) lower than the mean $\mathrm{V}$-MI score for the marketed control (percent reductions $=12.0 \%$ at week 2 , $12.2 \%$ at week 4 , and $11.1 \%$ at week 6 ). There were no statistically significant differences in V-MI scores between the polypyrophosphate groups.

There were a total of 15 minor adverse events reported by 13 subjects. All of these events were unremarkable and involved subjects in each of the 3 treatment groups. No subjects discontinued treatment due to an adverse event.

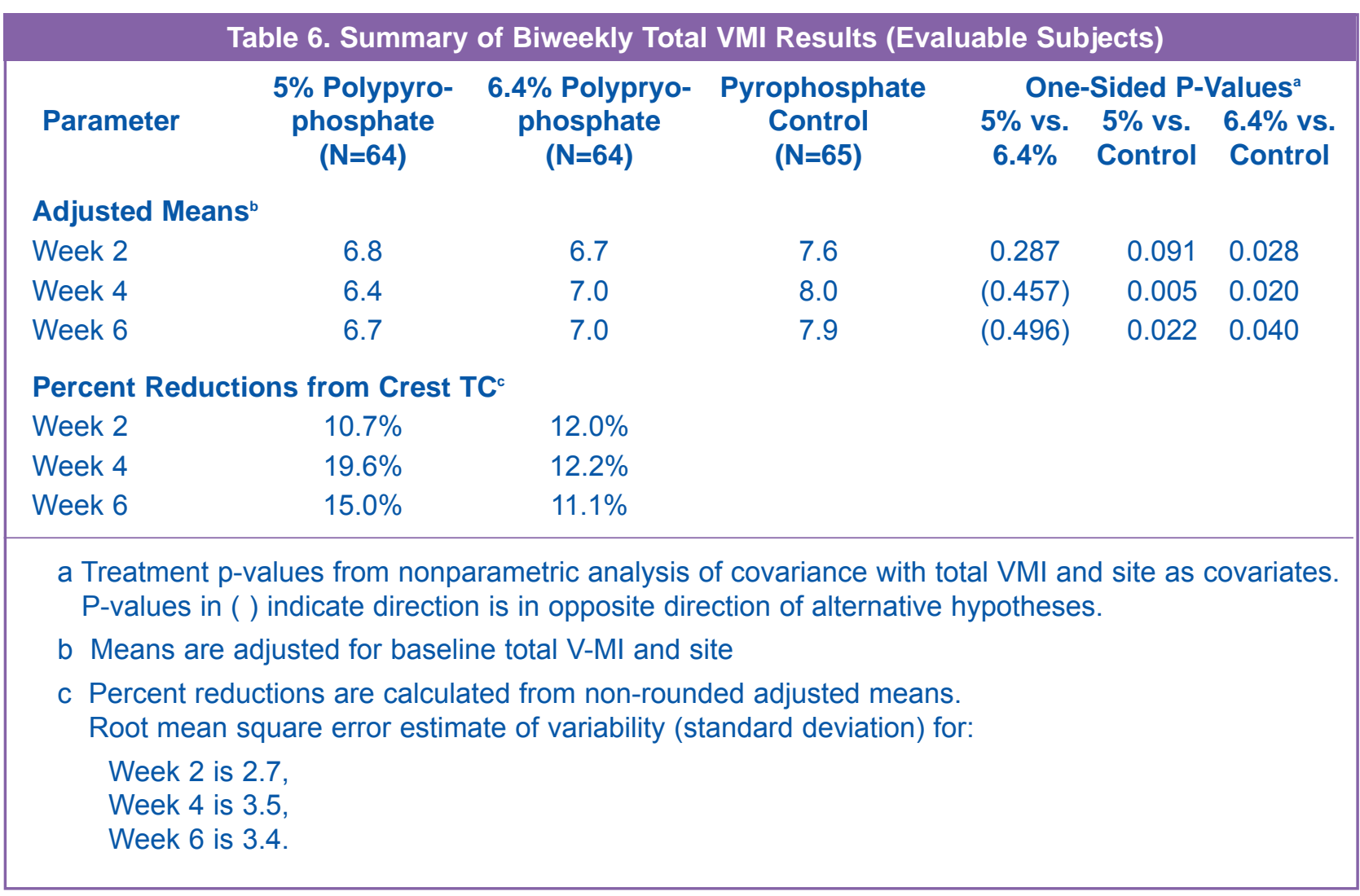




\section{Discussion}

Recent formulation and packaging advancements have allowed for development of a novel polypyrophosphate-containing dentifrice, Crest $^{\circledR}$ Multicare Advanced Cleaning (hereafter CMAC). The polymeric phosphate applied in CMAC is a Glass H 'hexametaphosphate,' a polymer of pyrophosphate with 10-12 repeating pyrophosphate subunits. CMAC was formulated at a concentration of $5 \%$ anion polymer, the equivalent anion concentration to pyrophosphate found in Crest Tartar Control. Also included in the formula is sodium fluoride (1100 ppm $\mathrm{F}$ as $\mathrm{NaF}$ ) for anticaries control and silica abrasive; these delivered at standard concentrations in a cost effective and functional dual phase package. Relative to pyrophosphate percursors, this polypyrophosphate dentifrice may offer significant clinical advantages with respect to extrinsic dental stain and calculus control efficacy. The relative merits of the polypyrophosphates was demonstrated in a "proof of concept" clinical trial. In this study, which used an accelerated calculus formation model to evaluate prevention of new calculus formation (the so-called "tartar control"), polypyrophosphate at two different concentrations was shown to exhibit superior efficacy relative to the current pyrophosphate dentifrice standard. These benefits (11-19\% incremental reductions compared to the industry tartar-control standard) were seen for both the $5 \%$ and $6.43 \%$ polypyrophosphate groups.Clinical response was impressive. Some subjects in the polypyrophosphate groups accumulated little-tono calculus during the treatment phase, despite using the modified brushing regimen for 6 weeks (Figures 10, 11).

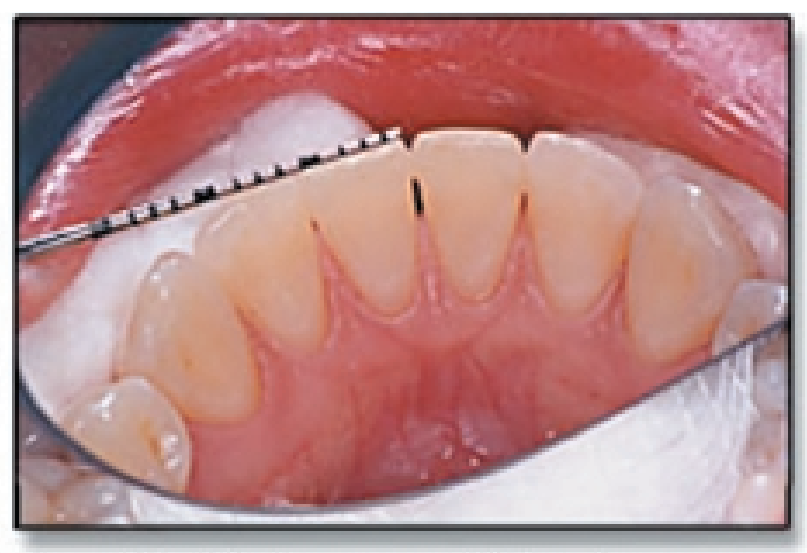

Figure 10. Calculus accumulation after 6 weeks using the modified, partial non-brushing regimen $5 \%$ polypyrophosphate

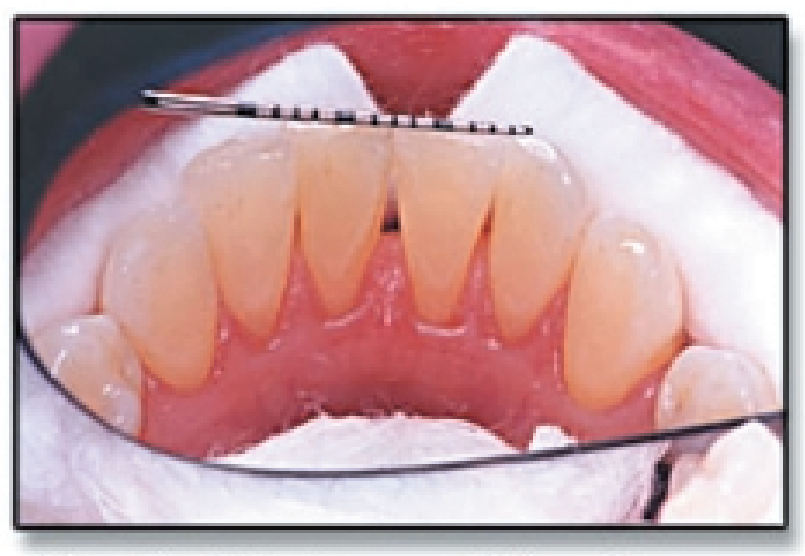

Figure 11. Calculus accumulation after 6 weeks using the modified, partial non-brushing regimen, $6.43 \%$ polypyrophosphate

This represents the first full report of the clinical response associated with this novel, dual-phase dentifrice. While this proof-of-concept study demonstrates the clinical merit of a $5 \%$ polypyrophosphate dentifrice formulation, additional research is needed to fully characterize the nature of the clinical response. Recently a series of preclinical and clinical studies have been completed on intraoral tolerability, stain control, and surface conditioning activity of the CMAC formulation as well as further investigations of relative anticalculus performance. This research is currently undergoing preparation for peerreviewed publication. The development of improved products and procedures often result from synergies of ideas and technologies that are apparently unrelated. We believe the development of CMAC represents one such case study. The coalescence of (1) improved technical understanding of factors important to dental surface cleaning, (2) dentifrice packaging permitting effective dual phase product executions, and (3) the safety and availability of polypyrophosphate have permitted the development of an improved cleaning dentifrice with improved clinical efficacy. We speculate the magnitude of these (whitening, tartar control, and clean conditioning) may be of sufficient magnitude and duration to produce meaningful changes in perception of hygiene effectiveness.

Studies also suggest that the polymer based system is better tolerated toward soft tissues than pyrophosphate analogue at similar dosages. ${ }^{21}$ Improved clinical efficacy and tolerability, along with conditioning signals, should encourage patient compliance with oral hygiene further 
complementing professional efforts directed at disease prevention. The incorporation of these cleaning and conditioning advantages into chemotherapeutic dentifrices can also be reasoned as essential, since the neglect of these important attributes likely limits the magnitude of chemotherapeutic ingredient actions in real clinical situations as compared to the controlled clinical trial settings. This continues to be an objective in our future research.

\section{Conclusion}

Innovations in the areas of tooth surface cleaning, packaging, and mechanism have contributed to the formulation of a novel, dual phase, polypyrophosphate dentifrice having superior anticalculus efficacy as well as other attributes that may contribute to clinical response and patient acceptability.

\section{References}

1. White DJ. Dental calculus: recent insights into occurrence, formation, prevention, removal and oral health effects of suprangingival and subgingival deposits. Eur J Oral Sci1997;105: 508-522.

2. White DJ. Tartar control dentifrices: current status and future prospects. In: Embery G, Rolla G, editors. Clinical and Biological Aspects of Dentifrices. Oxford: Oxford University Press, 1992: 277-291.

3. Rustogi KN, Triratana T, Lindhe J, Kietprajuk C, Volpe AR. The association between supragingival calculus deposits and the extent of gingival recession in a sample of Thai children and teenagers. J Clin Dent 1991; 6-11.

4. Mallatt ME, Beiswanger BB, Swancar JR, Stookey GK, Hennon DK. Influence of soluble pyrophosphate on calculus formation in adults. J Dent Res 1985;64: 1159-1162.

5. Zacherl WA, Pfeiffer HJ, Swancar JR. The effect of soluble pyrophosphates on dental calculus in adults. J Am Dent Assoc 1985;110: 737-738.

6. Hagmann M. A gene for smooth running joints. Science 2000; 289:226-227.

7. White DJ. Processes contributing to the formation of dental calculus. Biofouling 1991;4: 209-218.

8. Rowles SL. Composition of supragingival calculus. J Dent Res 1967;46: 114-119.

9. White DJ, Bowman WD, Nancollas GH. Physical chemical aspects of dental calculus formation and inhibition: in vitro and in vivo. In: ten Cate J, editor. Recent Advances in the Study of Dental Calculus. 1989: 175-188.

10. Sammons MC, Coombs MA, Deibel RM, Collier WG, McCormack L, White DJ. Dentifrice effects on uptake and retention of pyrophosphate in plaque. J Dent Res 1989;68: 421 Abstract.

11. Adams D. Calculus inhibition agents: A review of recent clinical trials. Adv Dental Res 1995;9: 410-418.

12. Stookey GK, Jackson RJ, Beiswanger BB, Stookey KR. Clinical efficacy of chemicals for calculus prevention. In: ten Cate J, editor. Recent Advances in the Study of Dental Calculus. Oxford: IRL Press, 1989: 235-258.

13. Volpe AR, Petrone ME, Davies R. A review of calculus clinical efficacy studies. J Clin Dent 1992; 4: 71-81.

14. Volpe AR, Manhold JH, Hazen SP. In vivo calculus assessment, part I: a method and its examiner reproducibility. J Periodontol 1965;36: 299-304.

15. Gaffar A, Polefka T, Afflitto J, Esposito A, Smith S. In vitro evaluations of pyrophosphate/copolymer/NaF as an anticalculus agent. Compend Supplt 1987; Suppl. 8: S242-250.

16. Rykke M, Rolla G. Desorption of acquired enamel pellicle in vivo by pryophosphate. Scand J Dent Res 1990;98: (3)211-214.

17. Rolla G, Rykke M, Sonju T. Effect of pyrophosphate on protein adsorption to hydroxyapatite in vitro and on pellicle formation in vivo. Scand J Dent Res 1988;96: 517-522. Abstract. 
18. Kozak KM, White DJ. Dentifrice effects toward chemical stain control: An in vitro comparison. J Dent Res 2000;79: 404 Abstract.

19. White DJ, Glandorf WM, McClanahan SF, Cox ER, Estes PD. Inventors. Dentifrice compositions containing polyphosphate and fluoride. 1999; (U.S. Patent \#5,939,052).

20. White DJ, Campbell SJ, Hyde JP, Gerlach RW. Novel model for evaluating anticalculus efficacy of tartar control dentifrices. J Dent Res 1999;78: 497 Abstract.

21. Gerlach RW. Comparative intraoral tolerance of polyphosphate and pyrophosphate antitartar dentifrices. J Dent Res 2000;79: 429 Abstract.

\section{About the Authors}

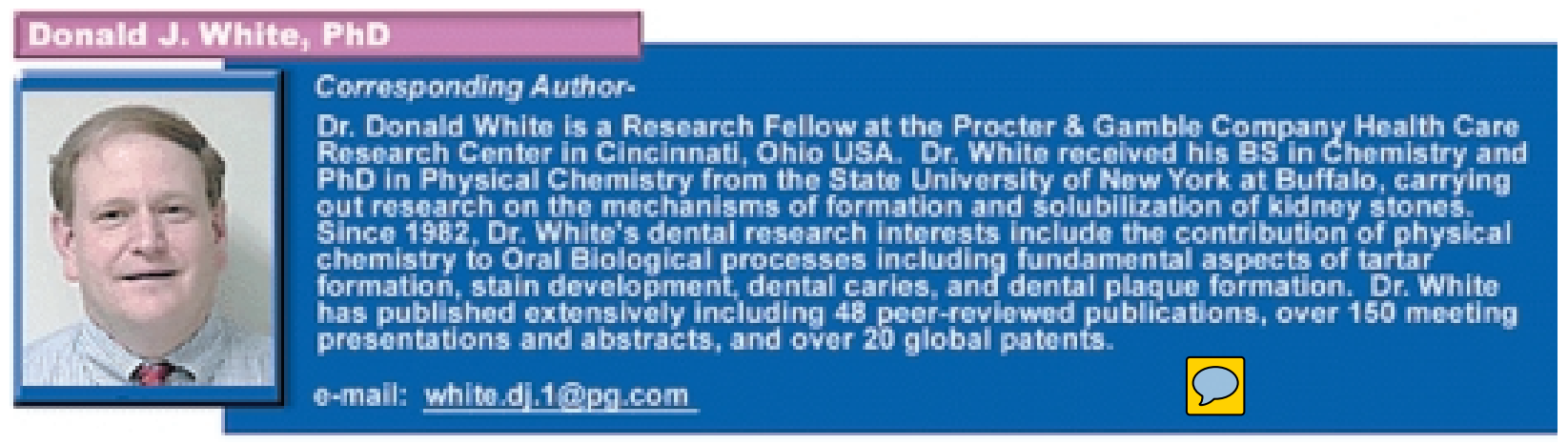

\section{Robert W. Gerlach, DDS, MPH}

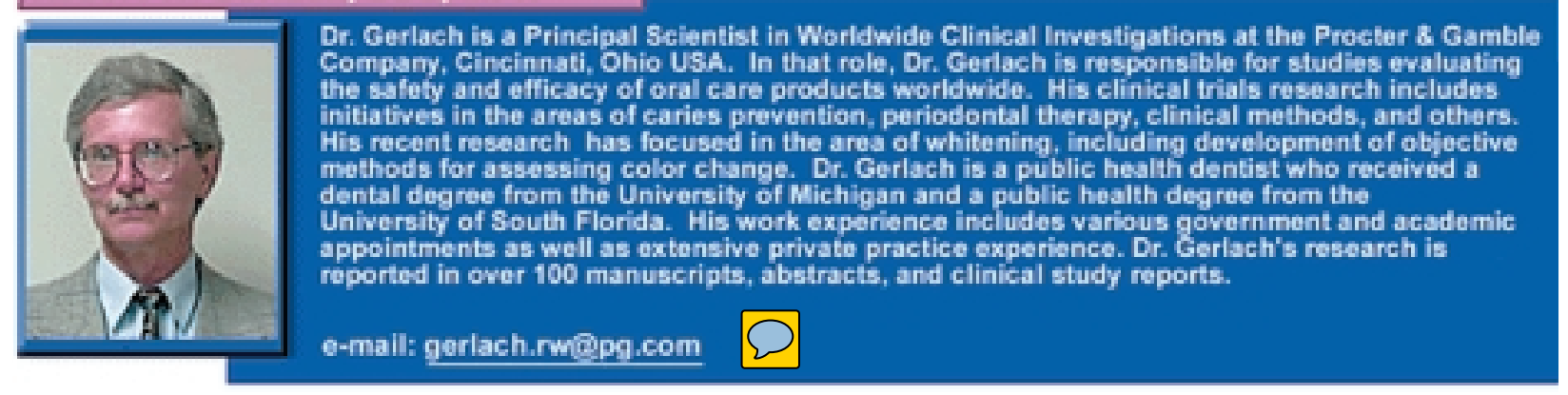

